\title{
Petite hydraulique : les actions de la Commission Européenne
}

\author{
Small hydro European Commission actions \\ par H. Pauwels \\ Commission Européenne -DG XVII- Technologie Energétique
}

White Paper " Energy for the Future : Renewable Sources of Energy" is the document which will lead the European policy for the promotion of these energies. Among the tools at disposal of the Cammission, the THERMIE programme for energy technologies development supports demonstration actions in various fields including Small-scale hydroelectric plants $(<10 \mathrm{MW}$ ). Since 1984, many positive results have been obtained. The Vth Framework for R.T.D. (1999-2002) should continue the ongoing actions to allow Small Hydro to continue to develop.

\section{I INTRODUCTION : LE LIVRE BLANC}

Le Livre Blanc "Energie pour l'Avenir : Les Sources d'Energie Renouvelables" est actuellement pour le développement des énergies renouvelables en général et pour la petite hydraulique en particulier, le document de base sur lequel s'appuieront les diverses initiatives politiques, législatives, administratives et techniques pour atteindre le but fixé dans ce même document : $12 \%$ d'énergies renouvelables dans la consommation eưropéenne d'énergie en 2010.

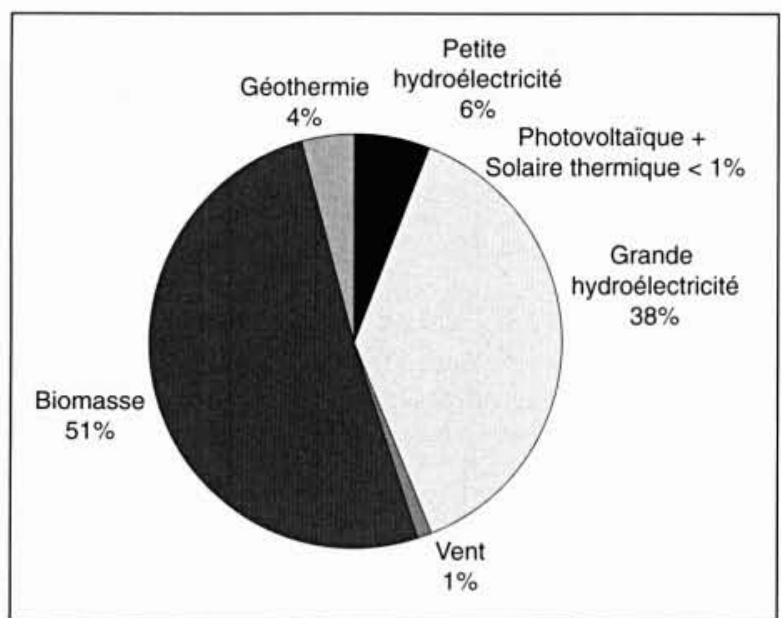

Production à partir de source d'énergie renouvelables en Europe en 1995

\section{II $\square$ PRINCIPALES CARACTÉRISTIQUES DU PLAN D'ACTION DU LIVRE BLANC}

Sans des efforts déterminés et coordonnés pour mobiliser les potentialités des énergies renouvelables dans I'Union, les objectifs fixés par le Livre Blanc ainsi que les engagements pris à Kyoto pour réduire les émissions de gaz à effet de serre ne pourront être atteints. Les moyens à mettre en œuvre pour réaliser ces objectifs sont au nombre de quatre :

1) Mesures relatives au marché intérieur, à savoir :

- un accès non discriminatoire au marché de l'électricité, - des mesures fiscales et financières, ainsi que des initiatives spécifiques à la biomasse et à l'énergie dans les bâtiments.

2) Renforcement des politiques communautaires La priorité donnée aux énergies renouvelables dans les politiques, les programmes et les budgets communautaires est actuellement faible. Il existe donc une grande marge de manœuvre pour renforcer leur présence. Ces renforcements visent tout particulièrement les politiques suivantes :

- Environnement ( $\mathrm{V}^{\circ}$ Programme d'action pour l'environnement),

- Croissance, Compétitivité et Emploi,

- Concurrence et aides d'Etat.

L'analyse des différents moyens disponibles pour promouvoir le développement des SER devrait prendre en compte les effets positifs de la concurrence. Il conviendrait donc de laisser jouer les forces du marché de manière à faire baisser rapidement les coûts de production de ces énergies.

LEXIQUE :

$\mathrm{ER}=$ Energie Renouvelable

SER $=$ Source d'Energie Renouvelable
$\mathrm{PH}=$ Petite Hydraulique

F.E.D. = Fonds Européen de Développement. 
Néanmoins, la Commission tiendra compte des dérogations relatives aux aides d'Etat. Le principe guide pour apprécier la compatibilité d'une aide en faveur des SER est que les effets bénéfiques de ces mesures sur l'environnement doivent compenser les effets de distorsion de la concurrence. - Recherche, Technologie, Développement et Démonstration. Il est notoire que de grandes potentialités existent pour la RTD\&D pour perfectionner les technologies, réduire les coûts et bénéficier du retour d'expériences des projets de démonstration. Le Ve programme-cadre, dont fait partie le successeur de THERMIE, se doit d'offrir plus de possibilités pour financer les efforts de RTD nécessaires dans ce domaine.

- Politique Régionale.

Les SER ont déjà leur place dans la politique régionale de l'Union. En 1999, des négociations devraient permettre d'étendre, de consolider et de clarifier les différentes aides possibles.

- Politique Agricole Commune et Politique de Développement rural ( $\mathrm{Ne}$ concerne que la biomasse).

\section{- Relations extérieures.}

La promotion des Energies Renouvelables dans les pays tiers est importante notamment pour contribuer à diminuer les émissions de $\mathrm{CO}_{2}$. Les programmes PHARE, TACIS, MEDA et les FED devraient donc mettre en avant les Energies Renouvelables. La politique de coopération et d'exportation en faveur des E.R. sera dynamisée en élargissant la portée et la base des programmes communautaires tels que SYNERGIE et les actions INCO du Ve programme-cadre.

3) Renforcement de la coopération entre Etats Membres

4) Mesures de soutien.

La promotion ciblée est réalisée par le programme ALTENER. D'autres mesures pour l'acceptabilité sur le marché et la protection du consommateur, l'amélioration de la position des sources d'énergie renouvelables sur le marché des capitaux des banques institutionnelles et commerciales ainsi que la création de réseaux d'information dans le domaine des S.E.R sont aussi prévues.

\section{III - CAMPAGNE POUR LE DÉCOLLAGE DES ÉNERGIES RENOUVELABLES}

L'objet de cette campagne est d'envoyer un message clair pour une utilisation plus extensive des sources d'énergie renouvelables. Cette campagne fera la promotion de projets d'envergure. Les actions-clés retenues sont :

- un million de systèmes photovoltaïques, soit 500000 systèmes de toitures et façades solaires pour le marché domestique et 500000 équipements pour les villages des pays en développement. Avec une moyenne de $1 \mathrm{~kW}$ par système, on compte ainsi installer $1000 \mathrm{MWe}$.

- $10000 \mathrm{MW}$ de grands parcs éoliens,

- 10000 MWth d'installations de biomasse,

- et finalement, l'intégration des énergies renouvelables dans 100 communautés, particulièrement de manière locale et dans des zones où l'on peut espérer une contribution de $100 \%$ et ce, pour une puissance totale de $1500 \mathrm{MW}$. La petite hydraulique (ainsi que la géothermie) ne fera pas directement partie de ces actions-clés, mais elle pourrait très bien se retrouver dans le programme d'intégration.

\section{IV — LA PETITE HYDRAULIQUE DANS LE LIVRE BLANC}

Actuellement, la part des Energies Renouvelables dans la consommation intérieure brute de l'Union Européenne représente environ $6 \%$, grande hydraulique comprise. La réalisation de l'objectif de $12 \%$ de pénétration dépend clairement du succès et de l'augmentation des diverses énergies renouvelables. Il est donc important de fixer des sous-objectifs pour chaque énergie renouvelable.

L'image donnée par le Livre Blanc sur l'industrie hydroélectrique est la suivante : " la technologie est fiable et mature et la production est depuis longtemps compétitive avec les autres sources d'énergie. Cependant, le potentiel technique et économique existant pour le développement de la grande hydraulique, soit a déjà été utilisé, soit est indisponible à cause des contraintes environnementales. A contrario, seulement $20 \%$ du potentiel économique pour la petite hydraulique a été exploité jusqu'à présent. De plus, il existe beaucoup de petites centrales hydrauliques à l'arrêt, souvent pour des raisons de coûts d'exploitation et de maintenance, de même que pour des raisons de coûts de rachat de la production, mais qui pourraient être réhabilitées pour un investissement limité. De plus, les industriels de l'Union dominent le marché mondial de l'équipement pour la petite hydraulique.

En 1995, approximativement 307 TWh d'énergie hydraulique ont été produites dans I'Union pour une puissance de $92 \mathrm{GW}$. La petite hydraulique (<10 MW) compte pour $10 \%$ de la puissance installée $(9,3 \mathrm{MW})$ et a produit $37 \mathrm{TWh}$. Une augmentation de $10 \%$ de la puissance installée de la grande hydraulique, soit $8500 \mathrm{MW}$ est réalisable. Une puissance installée supplémentaire de $4500 \mathrm{MW}$ pour la petite hydraulique est un objectif réaliste qui pourrait être réalisé dans un cadre réglementaire plus favorable ".[1]

Construire $4500 \mathrm{MW}$ sur 15 ans, cela représente annuellement $300 \mathrm{MW}$. Les chiffres, fournis par EUROSTAT, sont disponibles pour EUR 15 seulement pour 3 années, mais on estime que 2400 MWe ont été construits entre 1985 et 1995 , soit $240 \mathrm{MW}$ par an. Apparemment, l'objectif n'est donc pas très ambitieux, mais dans les conditions actuelles, c'est déjà un tour de force. Quoi qu'il en soit, si de réelles mesures d'incitation ne sont pas prises, il est probable que l'on n'atteigne pas l'objectif fixé.

\section{THERMIE}

\section{- 5.1 Identité}

Le programme JOULE-THERMIE est le programme spécifique pour la promotion et le développement des technologies énergétiques non nucléaires, au sein du IVe programme-cadre (1994-1998) pour la Recherche et la Développement Technologique. Le programme THERMIE en est la partie Démonstration. Pour les 4 ans du programme, un budget de 565 millions d'ECU a été attribué aux actions de type A (projets de démonstration) et B (mesures d'accompagnement des résultats). Pour les E.R., le budget est de 150,7 millions d'ECU (type A uniquement). En ce qui concerne la P.H., le montant de l'aide a été de près de 12 millions d'ECU.

\subsection{But}

L'objectif principal du programme vise l'amélioration de l'approvisionnement énergétique via des technologies plus efficaces. La réduction des conséquences néfastes sur l'envi- 
ronnement de la production et de la consommation d'énergie est une deuxième priorité du programme.

\subsection{Moyens}

Les projets de démonstration (Type A) bénéficient d'une contribution communautaire de $40 \%$ maximum et consistent en la réalisation concrète d'un projet. Les actions de Type B se présentent sous de multiples facettes mais consistent pour l'essentiel en des mesures préparatoires, d'accompagnement et de soutien des résultats du programme en amplifiant son impact et ses performances sur le marché.

\subsection{But du programme spécifique à la Petite Hydraulique}

Bien que les priorités définies dans l'Annexe Technique du Programme de Travail évoluent d'année en année, les lignes directrices sont restées identiques au cours du temps. Les objectifs visent à exploiter une plus grande variété de sites afin d'accroître la capacité de production, de réduire les coûts d'investissement, de production et d'exploitation et de réaliser des ensembles efficaces et fiables. Ces objectifs sont développés à travers deux axes : la rénovation/restauration et les projets sur site vierge. La priorité est accordée aux projets ayant un faible coût unitaire du kW installé ainsi qu'aux installations dont l'impact environnemental est minimum.

\section{- 5.5 Données}

Depuis 1984 , la Commission a reçu un total de 540 propositions. Les origines géographiques traduisent assez bien les zones où la petite hydraulique est bien implantée.

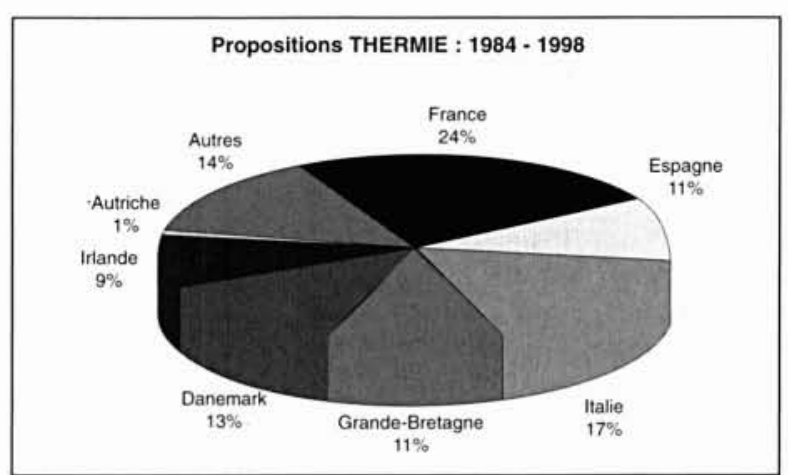

La France représente $25 \%$ des propositions, suivie par I'Italie avec $17 \%$. Après évaluation des propositions, 211 projets ont été retenus et soutenus par la Commission. Le coût total de ces projets est de près de 400 millions d'ECU pour lesquels la Commission a apporté une aide de 48,6 millions d'ECU, soit une moyenne de 12,3\%. La puissance totale installée des projets soutenus est de $169 \mathrm{MW}$, ce qui représente une aide moyenne de 288 ECU/kW. Parmi les 211 propositions retenues, 18 ont été retirées. Les motifs de ce retrait sont, en général, l'impossibilité d'obtenir des autorisations ou de réaliser le montage financier.

\section{- 5.6 Leçons tirées de la réalisation des projets}

L'importance et le type d'innovation des projets sélectionnés sont très variables. Les projets peuvent se classer en 2 catégories :

- les projets de démonstration d'une réalisation technologique au moyen de techniques innovantes, comme par exemple, l'application d'un matériau nouveau,

- les projets de démonstration d'un concept innovateur au moyen de technologies modernes, comme par exemple, l'intégration d'une turbine dans un réseau de distribution d'eau potable.

Ces deux catégories représentent respectivement $75 \%$ et $25 \%$ des projets.

Les domaines explorés par la démonstration concernent à peu près tout le champ d'activité de la P.H. et il est assez malaisé de dégager des tendances sur les projets terminés et en cours.

Les sites utilisés pour la démonstration ont les caractéristiques suivantes :

- puissance installée moyenne : $860 \mathrm{~kW}$, mais la médiane est de $440 \mathrm{~kW}$, ce qui indique une large population de petits projets,

- hauteur de chute moyenne : $47,8 \mathrm{~m}$, mais $50 \%$ des projets ont une hauteur inférieure à $10 \mathrm{~m}$.

Les domaines technologies abordés ont été les suivants :

- Equipement électromécanique (37\%) dont les matériaux nouveaux, le nouveau design d'organe, l'amélioration des caractéristiques hydrauliques, la standardisation,

- Electronique (20\%) dont l'optimisation du contrôle, les technologies de l'information (remplacement des opérateurs), l'électronique de puissance,

- Autres dont par exemple les centrales flottantes pour exploiter l'énergie cinétique, les machines à déplacement d'air.

Pour les 80 projets actuellement terminés, presque tous produisent de l'électricité mais pas toujours selon les conditions escomptées. Les véritables succès sont à peu près au nombre de 20. Il s'agit de projets ayant à la fois un bon degré d'innovation, ayant fait l'objet d'une bonne publicité et d'une commercialisation substantielle.

En général, la clé du succès se trouve dans l'association d'un fabricant de turbine ou d'une société d'ingénierie spécialisée avec le propriétaire du site qui finance le projet.

\section{- 5.7 Résultats de l'appel à proposition 1998}

Pour le dernier appel à proposition du IVe Programmecadre, 29 propositions ont été reçues. Elles représentent une puissance installée totale de $35,6 \mathrm{MW}$, un coût total de 21,6 millions d'ECU, soit un coût spécifique de $2100 \mathrm{ECU} / \mathrm{kW}$.

Les sept propositions sélectionnées recevront une aide globale de 1 million d'ECU.

Le champ d'action des 7 propositions se développe suivant 3 axes :

- Augmentation de la production par le développement de turbines plus adaptées aux conditions naturelles : Deriaz, Kaplan (hauteur de chute de $48 \mathrm{~m}$ ) et Stenzel,

- Rendre minimum l'impact sur l'environnement : 2 projets, - Utilisation combinée de l'eau et des structures préexistantes : 2 projets.

\section{- 5.8 Tendances futures}

Bien que des barrières au développement de la petite hydraulique soient essentiellement non technologiques, les projets démontrant des solutions d'intégration environnementale à un coût acceptable ainsi que les projets améliorant la compétitivité par l'abaissement des coûts (ex : simplification des turbines) ou l'augmentation de la production (ex. : vitesse variable) devraient constituer l'essentiel des développements dans le domaine. 


\section{Q PROGRAMME ALTENER}

\subsection{But}

Approuvé en 1992, le programme ALTENER a pour but de développer les sources d'énergie renouvelable dans I'Union et d'accroître le commerce des produits, des équipements et services au sein et à l'extérieur de l'Union.

\section{- 6.2 Moyens}

\section{ALTENER agit :}

- par la promotion du marché des ER et leur intégration dans le marché énergétique interne et ceci plus particulièrement au travers de mesures légales, de standardisation et spécifications techniques et par des études générales et sectorielles,

- par des mesures financières et économiques,

- par des activités de formation et d'information,

- et enfin, au travers de la coopération avec les pays tiers. En 1997, la Commission a adopté le programme ALTENER II qui poursuit les actions entreprises sous ALTENER dans un champ d'action élargi.

\subsection{Réalisations}

Une bonne douzaine d'actions typiquement Petite Hydraulique ont été ou sont actuellement aidées par le programme. Citons, par exemple, le "Layman's guide" en 2 volumes dont une version revue et augmentée est en cours de réalisation, ou bien encore un logiciel pour la réalisation de l'étude de faisabilité. Ce logiciel appelé PEACH permet d'obtenir facilement et avec une bonne précision, une évaluation de l'aspect "investissement" de l'aménagement. La réalisation d'un atlas informatisé des ressources hydroélectriques dans les différents pays de l'Union est toujours d'actualité. Dans le domaine de la formation, mentionnons l'initiative de I'Université de Grenoble qui propose un site de formation et d'essais.

Reste à préciser que les études normatives et pré-normatives sont inscrites dans le cadre du programme, mais que jusqu'à présent, aucune initiative venant du monde de la P.H. n'a été rencontrée.

\section{VII — AUTRE PROGRAMMES}

\section{VALOREN}

Mené dans le cadre de la politique régionale de développement, ce programme a permis, dans le passé, le développement de très nombreux sites au Portugal, en Espagne et en Italie.

\section{VIII — LE VE PROGRAMME-CADRE}

La continuation des actions entreprises dans les précédents programmes de technologie énergétique continuera dans le cadre du Ve Programme-cadre (1999-2003) pour la RTD. Après diverses propositions, la solution actuellement retenue est celle d'un programme spécifique Energie et Environnement : "Preserving the Ecosystem". Les détails du programme de travail sont en cours d'élaboration mais dès à présent, les Energies Renouvelables seront reprises à divers titres dans les 2 actions-clés concernant l'énergie.

\section{IX $\square$ CONCLUSIONS}

La Petite Hydraulique est spécifiquement identifiée et soutenue par deux programmes de la Communauté : THERMIE et ALTENER.

Les points faibles de la Petite Hydraulique dans les programmes de soutien sont probablement les suivants : l'image d'une énergie renouvelable qui n'est pas nouvelle, l'impact perçu comme négatif de l'implantation des centrales sur le milieu environnemental et, plus particulièrement pour les programmes technologiques, la maturité de la technologie qui exclurait toute innovation.

De manière plus générale, le manque de cohésion des acteurs de la Petite Hydraulique est probablement un point faible vis-à-vis de la politique de promotion qu'il faudrait mener.

Néanmoins, la Petite Hydraulique est et devrait rester l'une des composantes principales des E.R. pour atteindre l'objectif des $12 \%$.

L'impulsion donnée par la Livre Blanc et le suivi de Kyoto devraient amener des mesures pratiques de soutien.

\section{BIBLIOGRAPHIE}

[1] Energie pour l'Avenir: Les Sources d'Energie Renouvelables CB-CO97-676-FR-C 